\title{
TRANSMISSÃO VERTICAL DA SÍFILIS NA ATENÇÃO PRIMÁRIA: REVISÃO INTEGRATIVA
}

\author{
Gisélia de Moura Bezerra Cavalcanti \\ Lara Monteiro Costa Araújo " \\ Carla Laís dos Santos Fernandes " \\ Layza de Souza Chaves Deininger III
}

\section{RESUMO}

A Sífilis é uma doença infecciosa bacteriana transmitida por vias sexual e vertical. O objetivo deste trabalho é identificar os fatores que mantêm a sífilis gestacional, como problema de saúde pública no Brasil, por meio da produção científica. Trata-se de uma revisão integrativa, feita na Biblioteca Virtual em Saúde, no período de 2011 a 2019. Os resultados observaram que, mesmo em grávidas que cumpriram todo o pré-natal, o diagnóstico é tardio e a não realização, ou o tratamento inadequado das gestantes e dos parceiros são os principais responsáveis pela transmissão vertical, que pode ter consequências graves para o feto. Além disso, pode-se observar também que o não registro do tratamento na documentação da gestante e demora de resultados de exames, para constatação do diagnóstico, dificultam ainda mais sua realização adequada. Conclui-se que há falhas importantes no pré-natal, tanto no diagnóstico quanto no tratamento, levando a transmissão vertical da sífilis.

PALAVRAS-CHAVE: Gravidez. Sífilis. Transmissão Vertical de Doença Infecciosa.

Acadêmico do curso de Medicina da Faculdade de Ciências Médicas da Paraíba - FCM/PB. Departamento de Medicina. CEP: 58039-170. João Pessoa - Paraíba, Brasil. Autor Correspondente: giseliabcavalcanti1@hotmail.com. ORCID ID: https://orcid.org/0000-0002-5190-1234

Acadêmico do curso de Medicina da Faculdade de Medicina Nova Esperança - FAMENE. II Departamento de Medicina. CEP: 58067-695. João Pessoa - Paraíba, Brasil. ORCID ID: http://orcid.org/0000-0003-3144-7182; http://orcid.org/0000-0002-3687-205X

Enfermeira. Docente da Faculdade de Ciências Médicas da Paraíba - FCM/PB e Faculdade de Medicina Nova III Esperança - FAMENE. Departamento de Medicina. CEP: 58039-170. João Pessoa - Paraíba, Brasil. ORCID ID: http://orcid.org/0000-0001-5843-1805 


\section{INTRODUÇÃO}

A Sífilis é uma Doença Sexualmente Transmissível (DST), causada pela bactéria Treponema pallidum, a qual apresenta três fases: primária, secundária e terciária, com manifestações, tempo e consequências específicos em cada caso. ${ }^{1}$

O contágio da Sífilis ocorre por via sexual ou vertical, sendo essa última transmitida, durante a gestação, da mãe não tratada ou, inadequadamente tratada, para o filho, por disseminação hematogênica, através da placenta, constituindo a Sífilis Congênita (SC). ${ }^{1,2}$ A Transmissão Vertical pode acontecer em quaisquer fases da gestação e da doença, havendo maior probabilidade de infecção nas sífilis primária e secundária (50 a $100 \%)^{2}$. Associam-se à ocorrência de sífilis: baixo nível socioeconômico, coinfecção por HIV, uso de drogas, gravidez na adolescência, comportamento sexual de risco, acesso limitado aos cuidados de saúde e o não tratamento do parceiro infectado. ${ }^{3}$

O diagnóstico da doença é realizado pelo Teste Rápido Treponêmico para Sífilis nas seguintes situações especiais: localidades e serviços de saúde sem infraestrutura laboratorial ou regiões de difícil acesso; CTA - Centro de Testagem e Aconselhamento; segmentos populacionais mais vulneráveis às DSTs, de acordo com situação epidemiológica local; população indígena; gestantes e seus parceiros em unidades básicas de saúde, particularmente no âmbito da Rede Cegonha e outras situações especiais definidas pelo Departamento de DST, Aids e Hepatites Virais do Ministério da Saúde para ampliação do diagnóstico da sífilis. Todas essas descritas na Portaria $n^{\circ} 3.242$ ou, ainda, por Microscopia e Exames Sorológicos Treponêmicos ou NãoTreponêmicos nos outros casos, todos esses seguindo o Fluxograma Laboratorial para a
Sífilis. $^{4}$

Entre 1998 e 2018, segundo dados do Boletim Epidemiológico de Sífilis de 2018, do Ministério da Saúde, foram notificados no Sinan 188.445 casos de sífilis congênita em menores de um ano de idade. Durante o ano de 2017, 24.666 casos foram notificados, observandose uma taxa de incidência de 8,6 casos $/ 1.000$ nascidos vivos no Brasil. Isso é extremamente preocupante, pois mesmo sendo de fácil prevenção e com recursos disponíveis, inclusive na assistência pré-natal, a prevalência da doença permanece alta. Essa situação traduz as falhas na assistência à saúde prestada à gestante. ${ }^{5}$ Um dos principais determinantes na redução das taxas de transmissão vertical da sífilis é a qualidade da assistência à gestação e parto, tendo como fundamento do controle da doença a triagem sorológica e o tratamento adequado da gestante e parceiros. ${ }^{3}$

São considerados casos de sífilis na gestação: gestante com evidência clínica de sífilis e/ou com sorologia não treponêmica reagente, com qualquer titulagem, mesmo na ausência de resultado de teste treponêmico, realizada no pré-natal ou no momento do parto ou curetagem. ${ }^{2}$ Em 2017, foram notificados 49.013 casos de sífilis em gestantes, tendo uma taxa de detecção de 17,2 casos de sífilis em gestantes/1.000 nascidos vivos (28,4\% superior à taxa observada no ano anterior). Quanto aos Estados, o Rio de Janeiro teve a taxa de detecção mais elevada em todo o país, 35,6 casos/1.000 nascidos vivos, $34,5 \%$ em relação ao ano anterior, e Roraima, 7,6 casos/1.000 nascidos vivos, a menor, após queda de $35 \%$ quando comparado a $2016 . .^{5}$ Além das manifestações maternas, as consequências da doença para o feto são alarmantes, as quais incluem: parto prematuro, surdez, cegueira, deficiência mental e morte. ${ }^{1}$ Nos últimos dez anos, o coeficiente 
de mortalidade infantil por sífilis no Brasil passou de 2,3/1.000 nascidos vivos em 2007 para 7,2/1.000 nascidos vivos em 2017, o que corresponde a 206 óbitos por sífilis em crianças menores de um ano. 5

Diante desse aumento alarmante das taxas de infecção vertical da Sífilis, além de suas consequências na morbimortalidade infantil do país, o presente trabalho buscou identificar os fatores que mantêm a Sífilis Gestacional como problema de saúde pública no Brasil por meio de produção científica.

\section{MATERIAL E MÉTODOS}

Adotou-se a revisão integrativa como método de síntese do conhecimento acerca do tema proposto, com o intuito de responder à questão norteadora « Por que a sífilis gestacional é um problema de saúde pública no Brasil? >>.

A revisão foi realizada por meio das seguintes etapas: 1- identificação do problema de revisão, 2- seleção dos estudos, 3- extração dos dados dos estudos selecionados, 4avaliação dos estudos incluídos na revisão, 5- interpretação ou integração dos resultados, e 6-apresentação da revisão ou síntese do conhecimento presentes nos artigos analisados. Realizou-se uma revisão das publicações na área da saúde referente à transmissão vertical da Sífilis na Biblioteca
Virtual Bireme, buscando-se estudos nas bases de dados LILACS e MEDLINE. Com o intuito de obter informações mais atuais acerca do tema, preferiu-se produções científicas realizadas entre 2011 e 2019. Além disso, optou-se pelos termos dos Descritores em Ciências da Saúde (DeCs): Gravidez, Sífilis, Transmissão Vertical de Doença Infecciosa.

Os descritores foram aplicados de forma combinada, empregando o operador booleano AND: "Transmissão Vertical de Doença Infecciosa AND Sífilis", "Transmissão Vertical de Doença Infecciosa AND Gravidez" e "Sífilis AND Gravidez", envolvendo as bases de dados LILACS e MEDLINE, no idioma português, uma vez que o intuito foi analisar a literatura brasileira acerca do tema (Tabela 1).

TABELA 1: Seleção dos estudos de acordo com o uso da combinação dos descritores e base de dados pesquisada

\begin{tabular}{|c|c|c|c|c|}
\hline $\begin{array}{c}\text { Base de } \\
\text { dados }\end{array}$ & $\begin{array}{l}\text { Estudos } \\
\text { obtidos }\end{array}$ & $\begin{array}{l}\text { Estudos } \\
\text { excluídos }\end{array}$ & $\begin{array}{c}\text { Estudos } \\
\text { selecionados }\end{array}$ & Combinações de descritores \\
\hline \multirow{3}{*}{ MEDLINE } & 4 & 2 & " & $\begin{array}{l}\text { "Transmissão vertical da doença } \\
\text { infecciosa AND Sífilis" }\end{array}$ \\
\hline & 5 & 5 & 0 & $\begin{array}{c}\text { "Transmissão vertical da doença } \\
\text { infecciosa AND Gravidez" }\end{array}$ \\
\hline & 28 & 24 & 4 & "Sífilis AND Gravidez" \\
\hline Sub Total & 37 & 31 & 6 & - \\
\hline \multirow{3}{*}{ LILACS } & 14 & 12 & 2 & $\begin{array}{l}\text { "Transmissão vertical da doença } \\
\text { infecciosa AND Sífilis" }\end{array}$ \\
\hline & 39 & 37 & 2 & $\begin{array}{l}\text { "Transmissão vertical da doença } \\
\text { infecciosa AND Gravidez" }\end{array}$ \\
\hline & 123 & 117 & 6 & "Sífilis AND Gravidez" \\
\hline Sub Total & 176 & 166 & 10 & - \\
\hline Total & 213 & 197 & 16 & - \\
\hline
\end{tabular}


Os critérios de exclusão foram: (a) artigos anteriores a 2011,(b) ausência de resumo online disponível, (c) estudos duplicados, (d) publicações do tipo relato de caso, editorial, informes técnicos, (e) monografias, teses e dissertações, (f) fuga ao tema. Inicialmente, os resumos foram lidos com o intuito de verificar se apresentavam o enfoque buscado, posteriormente foram lidos na íntegra e excluídos conforme os critérios estabelecidos.

Foram encontrados 213 manuscritos que, após submetidos aos critérios de inclusão em uma seleção inicial, totalizou em 93 artigos. Posteriormente, os textos foram analisados e excluídos de acordo com os critérios préestabelecidos, obtendo-se 16 manuscritos na amostra final do estudo (Figura 1).

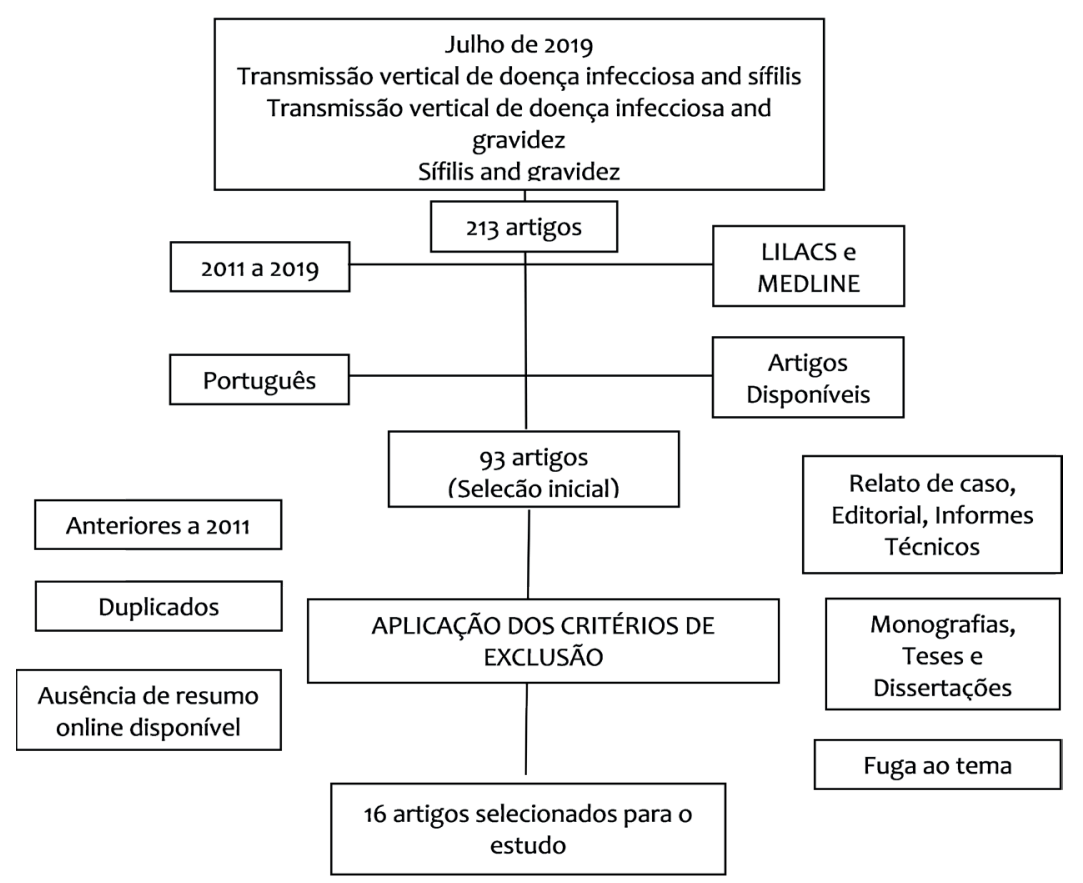

FIGURA 1. Fluxograma da pesquisa nas bases científicas

\section{RESULTADOS E DISCUSSÃO}

Após análise do material selecionado na literatura, optou-se pela divisão nas seguintes categorias: 1) Caracterização da sífilis gestacional e 2) Abordagem da sífilis no pré-natal pelos profissionais de Saúde.

Caracterização da sífilis gestacional

Foi constatado que em $85,7 \%$ a maioria das gestantes fizeram pré-natal, concluiu-se em $28,5 \%$ que o início do acompanhamento gestacional foi ainda no primeiro trimestre e, 14,2\%, no segundo trimestre. Em 57, $1 \%$ dos artigos, o diagnóstico foi realizado tardiamente no momento do parto. A maioria das gestantes não foram tratadas adequadamente $(42,8 \%)$ nem os parceiros (28,5\%). Quanto aos recém nascidos, em $28,5 \%$ dos estudos foi concluído que eles realizaram o VDRL (Venereal Disease Research 
Laboratory) ao nascer, em $28,5 \%$ foi possível analisar que os exames complementares não foram realizados e, em $14,2 \%$, eles não foram tratados. Em 14,2\%, mostrou os óbitos fetais causados pela sífilis. Constatou-se que nos estados do Amazonas, Ceará e Rio de Janeiro a maior proporção de casos de sífilis em gestantes se concentrou em residentes da capital, em contrapartida, no Espírito Santo e no Rio Grande do Sul havia mais casos em outros municípios (Tabela 2).

TABELA 2. Relação dos trabalhos selecionados com a caracterização da sífilis gestacional

\begin{tabular}{|c|c|c|c|c|}
\hline Autor & Ano & Título & $\begin{array}{c}\text { Revista/ } \\
\text { Base de dados } \\
\end{array}$ & Resultados \\
\hline Lafetá et al. & 2016 & $\begin{array}{l}\text { Sífilis materna } \\
\text { e congênita, } \\
\text { subnotificação e difícil } \\
\text { controle }\end{array}$ & $\begin{array}{l}\text { Rev. Bras. Epidemiol./ } \\
\text { LILACS }\end{array}$ & $\begin{array}{l}\text { A maioria das gestantes realizou corretamente o pré- } \\
\text { natal, porém o diagnóstico só foi realizado no parto, não } \\
\text { sendo tratadas. A maioria dos recém-nascidos fizeram } \\
\text { VDRL, sem exames complementares e foram tratados } \\
\text { adequadamente. Os casos foram subnotificados. }\end{array}$ \\
\hline Domingues et al. & 2016 & \begin{tabular}{l}
\multicolumn{1}{c}{ Incidência de } \\
sífilis congênita e \\
fatores associados à \\
transmissão vertical da \\
sífilis: dados do estudo \\
"Nascer no Brasil"
\end{tabular} & $\begin{array}{l}\text { Cad. Saúde Pública/ } \\
\text { LILACS }\end{array}$ & $\begin{array}{l}\text { A incidência de sífilis congênita foi } 3,51 \text { por mil nascidos } \\
\text { vivos, a taxa de transmissão foi } 34,3 \% \text {. Geralmente, as ges- } \\
\text { tantes realizaram pré-natal, iniciando no segundo trimes- } \\
\text { tre, com número de consultas inadequados e apenas um } \\
\text { VDRL. }\end{array}$ \\
\hline Holanda et al. & 2011 & $\begin{array}{l}\text { Perfil epidemiológico } \\
\text { da sífilis congênita no } \\
\text { Município do Natal, Rio } \\
\text { Grande do Norte - } 2004 \\
\text { a } 2007\end{array}$ & $\begin{array}{l}\text { Epidemiol. Serv. } \\
\text { Saúde/LILACS }\end{array}$ & $\begin{array}{l}\text { A taxa anual média de incidência de sífilis congênita foi } \\
6 \text { por mil nascidos vivos. As gestantes realizaram pré- } \\
\text { natal, de } 4 \text { a } 6 \text { consultas, com diagnóstico tardio no } \\
\text { parto, portanto, elas nem os parceiros foram tratados } \\
\text { adequadamente. A maioria dos recém-nascidos foi reativo } \\
\text { ao VDRL do sangue periférico. }\end{array}$ \\
\hline Fernandes et al. & 2014 & $\begin{array}{l}\text { Prevalência de HIV e } \\
\text { Sífilis em parturientes } \\
\text { atendidas em uma } \\
\text { maternidade de } \\
\text { referência na cidade de } \\
\text { Marabá-Pará } \\
\end{array}$ & $\begin{array}{l}\text { Revista Paraense de } \\
\text { Medicina/LILACS }\end{array}$ & $\begin{array}{l}\text { A prevalência estimada de sífilis materna foi } 1,9 \% \text {. Normal- } \\
\text { mente, as gestantes realizaram pré-natal, iniciando no pri- } \\
\text { meiro trimestre, com } 1 \text { a } 5 \text { consultas e diagnóstico tardio } \\
\text { no parto. }\end{array}$ \\
\hline Campos et al. & 2012 & $\begin{array}{l}\text { Sífilis em parturientes: } \\
\text { aspectos relacionados } \\
\text { ao parceiro sexual }\end{array}$ & $\begin{array}{l}\text { Rev. Bras. Ginecol. } \\
\text { Obstet./MEDLINE }\end{array}$ & $\begin{array}{l}\text { Os parceiros ficaram cientes do resultado positivo duran- } \\
\text { te o pré-natal. Em geral, as gestantes foram informadas } \\
\text { da importância do tratamento do parceiro, os quais se } \\
\text { trataram ( } 56 \% \text { ), mas o tratamento foi inadequado }(47,2 \%) \text {. }\end{array}$ \\
\hline Nascimento et al. & 2012 & $\begin{array}{l}\text { Gestações complicadas } \\
\text { por sífilis materna e } \\
\text { óbito fetal }\end{array}$ & $\begin{array}{l}\text { Rev. Bras. Ginecol. } \\
\text { Obstet./ MEDLINE }\end{array}$ & $\begin{array}{l}\text { A maioria das gestantes não possuíam antecedentes de } \\
\text { aborto, cesárea ou óbito fetal. Realizaram pré-natal, uma } \\
\text { ou duas consultas e o diagnóstico foi tardio. Na maioria } \\
\text { dos óbitos fetais, a única causa foi a sífilis. }\end{array}$ \\
\hline Kupek, Oliveira & 2012 & $\begin{array}{l}\text { Transmissão vertical } \\
\text { do HIV, da síflis e da } \\
\text { hepatite B no município } \\
\text { de maior incidência } \\
\text { de AIDS no Brasil: um } \\
\text { estudo populacional no } \\
\text { período de } 2002 \text { a } 2007 \\
\end{array}$ & $\begin{array}{l}\text { Rev. Bras. Epidemiol./ } \\
\text { LILACS }\end{array}$ & $\begin{array}{l}\text { A incidência de sífilis congênita foi 3,12 por mil nascidos } \\
\text { vivos. Na maioria dos casos de sífilis, as grávidas possuíam } \\
7 \text { ou mais consultas pré-natal, menos da metade recebeu } \\
\text { o tratamento preconizado. Nas gestantes inadequada- } \\
\text { mente, ou não tratadas ou parceiro não tratado, a trans- } \\
\text { missão vertical foi } 100 \% \text {. }\end{array}$ \\
\hline Saraceni et al. & 2017 & $\begin{array}{l}\text { Vigilância epidemi- } \\
\text { ológica da transmissão } \\
\text { vertical da sífilis: dados } \\
\text { de seis unidades federa- } \\
\quad \text { tivas no Brasil }\end{array}$ & $\begin{array}{l}\text { Rev. Panam. Salud. } \\
\text { Publica }\end{array}$ & $\begin{array}{l}\text { A taxa de incidência de sífilis congênita cresceu em quatro } \\
\text { estados e no Distrito Federal entre } 2007 \text { e } 2012 \text {, variando } \\
\text { de } 35,6 \% \text { no Distrito Federal a } 63,9 \% \text { no Rio Grande do Sul. } \\
\text { No Amazonas, houve redução de } 0,7 \% \text { na taxa de sífilis } \\
\text { congênita no período. }\end{array}$ \\
\hline
\end{tabular}


Abordagem da sífilis no pré-natal pelos profissionais de Saúde

Foi constatado em 50,0\% dos estudos que as gestantes não realizaram o pré-natal, ou o fizeram tardiamente. Em $62,5 \%$ dos artigos foi notado que não houve tratamento para os parceiros, poucos foram tratados, ou o fizeram de maneira incorreta, com 37,5\% destes constatando a dificuldade que existe em abordá-los. Em 12,5\% dos estudos, foi possível observar altas taxas de contaminação masculina. Em 37,5\% dos artigos foi apontado que havia diferenças entre o relato da gestante e o que constava na sua documentação, sendo necessária a repetição do tratamento. Em 12,5\% dos escritos foi abordado sobre a demora dos resultados dos exames. Em $25,0 \%$ foi relatado que os maiores índices de sífilis gestacional são em negras. Em 12,5\% dos estudos foi observado que a região Centro-Oeste é a que apresenta maiores taxas de sífilis na gestação. Dentro de $25,0 \%$ dos artigos coletados foi analisado que a sífilis congênita pode ser controlada com diagnóstico precoce, tratamento correto, tanto da gestante quanto do parceiro, e um bom pré-natal. Em 12,5\% destes foram observados índices menores dessa patologia em locais com Unidades de Saúde da Família (Tabela 3).

Constatou-se, em relação aos anos de publicação do material obtido, que $2(12,5 \%)$ foram publicados em 2011, sete (43,7\%) em 2012, três (18,7\%) em 2013 e um $(6,25 \%)$ em 2014, dois (12,5\%) em 2016, uma (6,25\%) em 2017. Nenhuma publicação de 2015, 2018 e 2019 foi selecionada. Quanto aos periódicos, os que mais exibiram publicações sobre o tema, deram ênfase em saúde pública, saúde coletiva e nas áreas de ginecologia e obstetrícia. São eles: Caderno de Saúde Pública com três estudos (18,7\%), a Revista de Saúde Pública, Revista Brasileira de Epidemiologia, Revista Brasileira de Ginecologia e Obstetrícia com dois artigos cada uma (12,5\%), Revista Brasileira de Saúde Materna Infantil,
Epidemiologia e serviços de Saúde, Ciências \& Saúde Coletiva, Revista Baiana de Saúde Pública, Revista Paraense de Medicina, DST - Jornal Brasileiro de Doenças Sexualmente Transmissíveis, Revista Panamericana de Salud Pública com um artigo cada $(6,25 \%)$.

Com base nos artigos selecionados, o perfil epidemiológico da sífilis gestacional é constituído predominantemente por gestantes com menos de 30 anos, solteiras ou com parceiros íntimos e escolaridade incompleta. A maior parte delas realizou o pré-natal no primeiro, ou segundo trimestre e com números de consultas que variou entre os estudos. Entretanto, mesmo quando o número de consultas atingiu o recomendado pelo Ministério da Saúde (MS), o diagnóstico não foi realizado, pois o mínimo de testes sorológicos para sífilis preconizados no pré-natal não foi obedecido. Recomenda-se a realização de três VDRL no pré-natal, porém observou-se que, na maioria dos casos, apenas um foi feito, sendo o diagnóstico realizado apenas no momento do parto, impossibilitando o tratamento da gestante e do parceiro. ${ }^{4,8}$

Normalmente, de acordo com dados estudados, os parceiros têm menos de 30 anos, relacionamento fixo com a gestante e são os pais da criança. Apesar de serem comunicados do diagnóstico ainda no pré-natal e se submeterem ao tratamento, a maior parte destes são considerados inadequadamente tratados. ${ }^{6}$ A transmissão vertical ocorreu em $100 \%$ dos casos e comprovou-se que gestante e o parceiro não foram tratados ou foram inadequadamente tratados. ${ }^{9} \mathrm{~A}$ incidência de sífilis congênita varia entre 3,12 casos a cada mil nascidos vivos ${ }^{9}$ e 3,51 casos para cada mil nascidos vivos7, comparando com o Canadá, que, entre 2010 e 2015, atingiu um percentual variante de 0,26 a 1,59 casos para cada 100.000 nascidos vivos, temos dimensão de como se trata de um problema de saúde pública no Brasil. ${ }^{23}$ 
TABELA 3. Relação dos trabalhos selecionados com a caracterização da sífilis gestacional

\begin{tabular}{|c|c|c|c|c|}
\hline Autor & Ano & Título & $\begin{array}{c}\text { Revista/ } \\
\text { Base de dados }\end{array}$ & Resultados \\
\hline Magalhães et al. & 2013 & $\begin{array}{l}\text { Sífilis materna e con- } \\
\text { gênita: ainda um desafio }\end{array}$ & $\begin{array}{l}\text { Caderno de Saúde } \\
\text { Pública/MEDLINE }\end{array}$ & $\begin{array}{l}\text { Ainda existem gestantes que não realizam o pré-natal. O } \\
\text { principal motivo da inadequação do tratamento de sífilis } \\
\text { em grávidas foi a ausência ou incorreto tratamento do } \\
\text { parceiro. Observou-se que algumas gestantes tiveram que } \\
\text { repetir o tratamento no momento do parto, por não pos- } \\
\text { suírem provas deste. }\end{array}$ \\
\hline Domingues et al. & 2013 & $\begin{array}{l}\text { Manejo da sífilis na } \\
\text { gestação: conhecimen- } \\
\text { tos, práticas e atitudes } \\
\text { dos profissionais } \\
\text { pré-natalistas da rede } \\
\text { SUS do município do Rio } \\
\text { de Janeiro } \\
\end{array}$ & $\begin{array}{l}\text { Ciênc. Saúde Coletival } \\
\text { MEDLINE }\end{array}$ & $\begin{array}{l}\text { Os profissionais de saúde afirmam que suas maiores } \\
\text { dificuldades com relação a sífilis gestacional, são: início } \\
\text { tardio do pré-natal, dificuldade de abordar os parceiros, } \\
\text { demora nos resultados dos testes para a doença, falta de } \\
\text { acesso ao teste treponêmico e a não adesão das gestantes }\end{array}$ \\
\hline Domingues et al. & 2013 & $\begin{array}{l}\text { Ś́filis congênita: evento } \\
\text { sentinela da qualidade } \\
\text { da assistência pré-natal }\end{array}$ & $\begin{array}{l}\text { Rev. Saúde Pública/ } \\
\text { LILACS }\end{array}$ & $\begin{array}{l}\text { Notou-se uma maior prevalência de síflis gestacional } \\
\text { em negras, com baixa escolaridade e início tardio do } \\
\text { pré-natal. Houve incompatibilidade entre o relato da ges- } \\
\text { tante e o documentado no cartão gestacional. Não foi reg- } \\
\text { istrado tratamento para parceiros. }\end{array}$ \\
\hline Domingues et al. & 2012 & $\begin{array}{l}\text { Avaliação das ações } \\
\text { de controle da sífilis e } \\
\text { do HIV na assistência } \\
\text { pré-natal da rede pública } \\
\text { do município do Rio de } \\
\text { Janeiro, Brasil }\end{array}$ & $\begin{array}{l}\text { Rev. Bras. Saúde Ma- } \\
\text { ter. Infant./LILACS }\end{array}$ & $\begin{array}{l}\text { Algumas gestantes não sabiam informar sobre VDRL ou } \\
\text { sífilis e não foram informadas desse diagnóstico. Trata- } \\
\text { mentos foram realizados de modo incorreto. Observou-se } \\
\text { que dificuldades encontradas no manejo da doença estão } \\
\text { relacionadas ao início tardio do pré-natal, demora dos re- } \\
\text { sultados dos exames e não abordagem de parceiros. }\end{array}$ \\
\hline Mesquita et al. & 2012 & $\begin{array}{l}\text { Análise dos Casos de Sífi- } \\
\text { lis Congênita em Sobral, } \\
\text { Ceará: Contribuições } \\
\text { para Assistência } \\
\text { Pré-Natal }\end{array}$ & $\begin{array}{l}\text { DST - J Bras Doenças } \\
\text { Sex Transm/LILACS }\end{array}$ & $\begin{array}{l}\text { A sífilis congênita pode ser controlada com diagnóstico } \\
\text { precoce e tratamento coerente, através de consultas } \\
\text { pré-natais, abordagem concreta do parceiro e presença } \\
\text { do mesmo nas consultas. Importante dividir as consultas } \\
\text { por toda a gestação e solicitar os exames nos trimestres } \\
\text { adequados. }\end{array}$ \\
\hline Araújo et al. & 2012 & $\begin{array}{l}\text { Incidência da sífilis } \\
\text { congênita no Brasil e sua } \\
\text { relação com a Estratégia } \\
\text { Saúde da Família }\end{array}$ & $\begin{array}{l}\text { Rev. Saúde Pública/ } \\
\text { LILACS }\end{array}$ & $\begin{array}{l}\text { Foi relatada tendência de queda nos índices de sífilis } \\
\text { congênita em locais abarcados pela Estratégia de Saúde } \\
\text { da Família. Poucos parceiros tratados após o diagnóstico } \\
\text { materno. Gestantes negras apresentaram maiores índices } \\
\text { sem realização do pré-natal, menor acesso ao diagnóstico } \\
\text { de sífilis e altas taxas de parceiros não tratados. }\end{array}$ \\
\hline Saraceni, Miranda & 2012 & $\begin{array}{l}\text { Relação entre a cober- } \\
\text { tura da Estratégia Saúde } \\
\text { da Família e o diagnósti- } \\
\text { co de sífilis na gestação } \\
\quad \text { e sífilis congênita }\end{array}$ & $\begin{array}{l}\text { Cad. Saúde Pública/ } \\
\text { MEDLINE }\end{array}$ & $\begin{array}{l}\text { A média nacional de sífilis gestacional gira em torno de } \\
1,6 \% \text {. A região centro-oeste foi a que apresentou maior } \\
\text { razão observada/estimada para sífilis na gestação (38\%). }\end{array}$ \\
\hline Costa et al. & 2011 & $\begin{array}{l}\text { HIV/AIDS e sífilis entre } \\
\text { gestantes adolescentes } \\
\text { e adultas jovens: fatores } \\
\text { de exposição e risco dos } \\
\text { atendimentos de um } \\
\text { programa de DST/HIV/ } \\
\text { AIDS na rede pública de } \\
\text { saúde/SUS, Bahia, Brasil. }\end{array}$ & $\begin{array}{l}\text { Revista Baiana de } \\
\text { Saúde Pública/LILACS }\end{array}$ & $\begin{array}{l}\text { Observou-se } 507 \text { resultados positivos para sífilis, sendo } \\
\text { destes } 324 \text { mulheres, com prevalência de } 1,5 \% \text { entre as } \\
\text { gestantes estudadas, notando-se o alto índice de contam- } \\
\text { inação masculina. }\end{array}$ \\
\hline
\end{tabular}


Os recém-nascidos de mães sifilíticas geralmente nascem com mais de 37 semanas, assintomáticos e com peso Adequado para Idade Gestacional (AIG). Na maior parte dos casos, testou-se o VDRL do sangue periférico, sendo este reativo, porém ignoraram-se os dados, ou não foram realizados exames complementares, como VDRL no líquor e raio-x de ossos longos. 4,5

A sífilis gestacional pode ocasionar desfechos desfavoráveis como óbito fetal ou neonatal, baixopesoaonascer, prematuridade, internação em UTI neonatal, dentre outros. ${ }^{7}$ Em um estudo realizado acerca do óbito fetal, foi observado que normalmente as grávidas tiveram algum acompanhamento pré-natal, mas metade delas compareceram a uma ou duas consultas, não sendo diagnosticadas. Além disso, não tinham antecedentes de aborto, cesárea ou óbito fetal. ${ }^{10} \mathrm{O}$ momento de admissão hospitalar foi realizado entre 28 e 36 semanas de gestação, com presença de trabalho de parto, porém sem batimentos cardiofetais. Na maioria dos óbitos fetais, a única causa clinicamente detectada relacionada foi a presença de sífilis.

Apesar da notificação compulsória, apenas $6,5 \%$ dos casos de sífilis gestacional e $24,1 \%$ de sífilis congênita foram notificados. ${ }^{4}$ Em relação as regiões brasileiras, a região Centro-Oeste tem o maior número de diagnósticos e de notificações da sífilis congênita. 18

Portanto, observa-se que o pré-natal não está sendo realizado adequadamente, uma vez que as gestantes aderem ao mesmo, mas não são diagnosticadas precocemente ou tratadas de forma adequada, assim como seus parceiros. Além disso, a assistência aos recémnascidos não está obedecendo a necessidade de exames complementares para sífilis. $\mathrm{Na}$ cidade de Nova lorque, aproximadamente $88 \%$ das gestantes infectadas por sífilis entre 2010 e 2016 não a transmitiram para os seus filhos, presumivelmente por causa do rastreamento e tratamento precoces, demonstrando o papel crítico que os provedores e sistemas de saúde pública desempenham na prevenção desta enfermidade. ${ }^{22}$

Em relação a abordagem da sífilis no pré-natal pelos profissionais de Saúde, estudos apontaram a existência de puérperas que não realizaram o pré-natal. Mesmo sendo uma minoria, impede o diagnóstico da sífilis e, portanto, a abordagem dos profissionais de saúde. ${ }^{11,16}$

Foi demonstrado que os trabalhadores da área de saúde relatam obstáculos na abordagem da sífilis, devido as gestantes iniciarem tardiamente o pré-natal, pelo não acompanhamento dos parceiros as consultas e pela dificuldade dos resultados dos exames, ou falta de acesso aos exames diagnósticos. ${ }^{12,14,17}$ Muitos tratamentos podem ser considerados inadequados por alguns profissionais não buscarem tratar também os parceiros. ${ }^{13,14}$ Além disso, podese concluir também, através da análise dos escritos que, com frequência, os profissionais prescrevem o segundo exame de sífilis para gestantes em épocas impróprias ao preconizado. Observou-se ainda que grande parte dos profissionais têm acesso aos manuais do Ministério da Saúde, que guiam sua abordagem, mas aproximadamente metade destes não os leem. ${ }^{11,15}$

Profissionais de saúde também lidam com a problemática das gestantes perderem a documentação que relata tratamento de sífilis, ou não estar corretamente preenchida, havendo, em alguns casos, diferenças entre o que consta nos documentos e o que relata a paciente, que, na maioria das vezes, não é informada sobre a forma clínica da sífilis. Foi possível observar também, que as orientações para uso de preservativos nas relações sexuais e riscos de infecção do feto não se integrava nos cartões das gestantes, inexistindo 
um local para esse dado. ${ }^{11,14}$ Estudos analisados mostraram que muitos parceiros se negam a utilizar preservativos, por afirmarem fidelidade, e as gestantes relataram constrangimento ao pedir aos parceiros para fazerem uso desse método. Ainda foi observado que algumas puérperas afirmam não saber o que é o VDRL nem a própria sífilis, e não sabem se esse exame foi solicitado. ${ }^{13,14}$ Além disso, notou-se que gestantes negras apresentam maiores índices de não realização do pré-natal, com pouco acesso ao diagnóstico da sífilis, devido a não comparecimento em consultas. 13,16

Com isso, foram percebidas

\section{CONSIDERAÇÕES FINAIS}

Asífilis gestacional éum grave problema de saúde pública devido a alta prevalência e grande desconhecimento das gestantes, dos parceiros e até mesmo pela falha na assistência à Saúde.

Portanto, é necessária a sensibilização dos profissionais de saúde e a melhoria na qualidade da assistência prestada durante pré-natal. Assim, é fundamental a função da Atenção Básica no intuito da captação precoce fragilidades no acompanhamento do pré-natal, uma vez que, mesmo com a adesão da maioria das gestantes, as quais realizam o mínimo de consultas preconizadas pelo Ministério da Saúde, os exames e os tratamentos não são realizados no período correto nem de forma adequada. Contudo, normalmente o diagnóstico de sífilis na gestante é realizado tardiamente, ou até mesmo no momento do parto, impossibilitando o tratamento e, consequentemente, ocorrendo a transmissão vertical da doença. Além disso, os parceiros geralmente são tratados de forma inadequada, ou não realizam o tratamento por, muitas vezes, não serem informados da necessidade.

das gestantes para que o pré-natal seja iniciado nos primeiros meses e, com isso, a realização do diagnóstico o quanto antes.

Ao detectar a doença, os profissionais devem incentivar as gestantes a realizarem o tratamento completo, assim como o de seus parceiros. As ações educativas devem ser encorajadas com o intuito de conscientizar a população a se prevenir. 


\title{
VERTICAL SYPHILIS TRANSMISSION IN PRIMARY ATTENTION: INTEGRATIVE REVIEW
}

\begin{abstract}
Syphilis is a bacterial infectious disease transmitted by sexual and vertical routes. The objective is to review the scientific production of vertical syphilis transmission. This is an integrative review carried out in the Virtual Health Library, from 2011 to 2016. The results observed that even in pregnant women who undergo prenatal care, the diagnosis is late, and the failure to perform or inadequate treatment of pregnant women and partners are primarily responsible for vertical transmission, which can have severe consequences for the fetus. Also, we can observe the non-registration of the treatment in the documentation of the pregnant woman and delay of results of exams to verify the diagnosis, making it even more difficult to perform it properly. It is concluded that there are essential failures in prenatal care, both in diagnosis and in treatment, leading to the vertical transmission of syphilis.
\end{abstract}

KEYWORDS: Pregnancy. Syphilis. Infectious Disease Transmission Vertical.

\section{REFERÊNCIAS}

1. Brasil. Ministério da Saúde (MS). PROTOCOLO PARA A PREVENÇÃO DE TRANSMISSÃO VERTICAL DE HIV E SÍFILIS. Brasília-DF. 2007. Disponível em: http://www.aids.gov.br/sites/default/ files/protocolo-bolsoo2web.pdf.

\section{SÍFILIS CONGÊNITA E SÍFILIS NA GESTAÇÃO.} Ver. Saúde Pública 2008;42(4):768-72. São Paulo-SP. Disponível em: http://www.scielo.br/pdf/ rsp/v42n4/itss.pdf.

3. Magalhães DMS, Kawaguchi IAL, Dias Al, Calderon IMP. SÍFILIS MATERNA E CONGÊNITA: AINDA UM DESAFIO. Cad. De Saúde Pública 2013. Disponível em: https://www.scielosp.org/scielo. php?pid=S0102-311X2013001000008\&script=sci_arttext\&tlng=pt .

4. Brasil. Ministério da Saúde. PORTARIA N ${ }^{\circ}$ 3.242 DE DEZEMBRO DE 2011. Gabinete do Ministro. 20122 Jan. Disponível em: http://www.aids. gov.br/sites/default/files/anexos/page/2010/233/ portaria_3242_12_pdf_28838.pdf.
5. BOLETIM EPIDEMIOLÓGICO - SÍFILIS ANO 2018. Secretaria de Vigilância em Saúde, Ministério da Saúde (MS). 2018 49(45). Disponível em: http://www.aids.gov.br/pt-br/tags/publicacoes/ boletim-de-sifilis.

6. Lafetá KRG, Martelli Júnior H, Silveira MF, Paranaíba LMR. Sífilis materna e congênita, subnotificação e difícil controle. São Paulo SP. Revista Brasileira de Epidemiologia. 2016 Jan/Mar 19(1). Disponível em: http://www.scielosp.org/scielo.php?script=sci_arttext\&pid=S1415-790X2016000100063\#aff1.

7. Holanda MTCG, Barreto MA, Machado KMM, Pereira RC. Perfil epidemiológico da síflis congênita no Município do Natal, Rio Grande do Norte - 2004 a 2007. Brasília - DF. Epidemiologia e Serviço de Saúde. 2011 Jun 20(2). Disponível em: http://scielo.iec.pa.gov.br/scielo.php?script=sci_arttex49742011000200009\&lng=pt\&nrm=iso\&tlng=pt. 
8. Campos ALA, Araújo MAL, Melo SP, Andrade RFV, Gonçalves MLC. Sífilis em parturientes: aspectos relacionados ao parceiro sexual. Rio de Janeiro - RJ. Revista Brasileira de Ginecologia e Obstetrícia. 201234 (9). Disponível em: http://www.scielo.br/scielo.php?scrip$\mathrm{t}=\mathrm{sci}$ _arttext\&nrm=iso\&lng=pt\&tlng=pt\&pi$\mathrm{d}=$ So100-72032012000900002.

9. Domingues RMSM, Leal MC. Incidência de sífilis congênita e fatores associados à transmissão vertical da sífilis: dados do estudo Nascer no Brasil. Rio de Janeiro - RJ. Caderno de Saúde Pública. 2016 32(6). Disponível em: http://www. scielosp.org/scielo.php?script=sci_arttext\&pi$d=$ S0102-311X2016000605002.

10. Fernandes HD, Araújo EC, Neves DCO, Ribeiro KTS. Prevalência de HIV e Sífilis em parturientes atendidas em uma maternidade de referência na cidade de Marabá-Pará. Revista Paraense de Medicina. 2014 Jul-Set 28(3). Disponível em: http://files.bvs.br/upload/S/0101-5907/2014/ v28n3/a4531.

11. Kupek E, Oliveira JF. Transmissão vertical do HIV, da sífilis e da hepatite B no município de maior incidência de AIDS no Brasil: um estudo populacional no período de 2002 a 2007. São Paulo - SP. Revista Brasileira de Epidemiologia. 2012 15(16). Disponível em: http://www.scielo. br/scielo.php?script=sci_arttext\&nrm=iso\&lng=pt\&tlng=pt\&pid=S1415-790X2012000300004.

12. Nascimento MI, Cunha AA, Guimarães EV, Alvarez FS, Oliveira SRSM, Bôas ELV. Gestações complicadas por sífilis materna e óbito fetal. Rio de Janeiro - RJ. Revista Brasileira de Ginecologia e Obstetrícia. 2012 34(2). Disponível em: http://www.scielo.br/scielo.php?scrip$\mathrm{t}=\mathrm{sci} \_$arttext\&nrm=iso\&lng=pt\&tlng=pt\&pi$\mathrm{d}=$ S0100-72032012000200003.

13. Magalhães DMS, Kawaguchi IAL, Dias A,
Calderon IMP. Sífilis materna e congênita: ainda um desafio. Rio de Janeiro - RJ. Caderno de Saúde Pública. 2013 29(6). Disponível em: http://www.scielo.br/scielo.php?script=sci_arttext\&nrm=iso\&lng=pt\&tlng=pt\&pid=S0102-311X2013000600008.

14. Domingues RMSM, Lauria LM, Saraceni $V$, Leal MC. Manejo da sífilis na gestação: conhecimentos, práticas e atitudes dos profissionais pré-natalistas da rede SUS do município do Rio de Janeiro. Rio de Janeiro - RJ. Ciência e Saúde Coletiva. 2018 18(5). Disponível em: http://www.scielo.br/scielo.php?script=sci_arttext\&nrm=iso\&lng=pt\&tlng=pt\&pid=S1413-81232013000500019.

15. Domingues RMSM, Saracen M, Hartz ZMA, Leal MC. Sífilis congênita: evento sentinela da qualidade da assistência pré-natal. São Paulo-SP. Revista de Saúde Pública. 2013 47(1). Disponível em: http://www.scielosp.org/scielo.php?scrip$\mathrm{t}=\mathrm{sci}$ _arttext\&pid=S0034-89102013000100019.

16. Domingues RMSM, Hartz ZMA, Leal MC. Avaliação das ações de controle da sífilis e do HIV na assistência pré-natal da rede pública do município do Rio de Janeiro, Brasil. Recife - PE. Revista Brasileira de Saúde Materno Infantil. 2012 12(3). Disponível em: http://www. scielo.br/scielo.php?script=sci_arttext\&pi$\mathrm{d}=$ S1519-38292012000300007.

17. Mesquita KO, Lima GK, Filgueira AA, Flôr SMC, Freitas CASL, Linhares MSC, et al. Análise dos Casos de Sífilis Congênita em Sobral, Ceará: Contribuições para Assistência Pré-Natal. DST - J bras Doenças Sex Transm. 2012 24(1). Disponível em: http://www.dst.uff.br/revista24-1-2012/7. Analise\%2odos\%20Casos\%20de\%20Sifilis\%20Congenita.pdf.

18. Araújo CL, Shimizu HE, Sousa AIA, Hamann EM. Incidência da sífilis congênita no Brasil e sua relação com a Estratégia Saúde da 
Família. São Paulo - SP. Revista de Saúde Pública. 2012 46(3). Disponível em: http://www. scielosp.org/scielo.php?script=sci_arttext\&pi$d=$ S0034-89102012000300010.

19. Costa MCO, Santos BC, Souza KEP, Cruz NLA, Santana MC, Nascimento OC. HIV/AIDS e Sífilis entre gestantes adolescentes e adultas jovens: Fatores de exposição e risco dos atendimentos de um programa de DST/HIV/AIDS na rede pública de saúde/SUS, Bahia, Brasil. Revista Baiana de Saúde Pública. 2011 35(1). Disponível em: http:// files.bvs.br/upload/S/0100-0233/2011/v35nSupl1/ a2308.pdf .

20. Saraceni V, Miranda AE. Relação entre a cobertura da Estratégia Saúde da Família e o diagnóstico de sífilis na gestação e sífilis congênita. Rio de Janeiro - RJ. Cadernos de Saúde Pública. 2012 28(3). Disponível em: http://www. scielo.br/scielo.php?script=sci_arttext\&pid=S0102-311X2012000300009. 21. Saraceni V, Pe- reira GFM, Silveira MF, Araújo MAL, Miranda $A E$. Vigilância epidemiológica da transmissão vertical da sífilis: dados de seis unidades federativas no Brasil. Rio de Janeiro - RJ. Revista Panamericana de Salud Pública. 2017 41:e44. Disponível em: https://scielosp.org/scielo.php?script=sci_arttext\&nrm=iso\&lng=pt\&tlng=pt\&pi$\mathrm{d}=$ S1020-49892017000100252.

22. Slutsker JS, Hennessy RR, Schillinger JA. Factors Contributing to Congenital Syphilis Cases New York City, 2010-2016. New York. Morbidity and Mortality Weekly Report. 2018 67(39). Disponível em: https://www.ncbi.nlm.nih.gov/pmc/ articles/PMC6171893/

23. Choudhri Y, Miller J, Sandhu J, Leon A, Aho J. Infectious and congenital syphilis in Canada, 2010-2015. Ottawa. Canada Communicable Disease Report. 2018 44(2). Disponível: https://www. ncbi.nlm.nih.gov/pmc/articles/PMC5864261/. 